Hoffmann-La Roche Ltd, Basel, Switzerland; ${ }^{2}$ UCL Institute of Child Health and Great Ormond Street Hospital, London, United Kingdom; ${ }^{3}$ Alder Hey Children's Hospital, Liverpool, United Kingdom; ${ }^{4}$ University of Utah Primary Children's Hospital, Salt Lake City, United States of America; ${ }^{5}$ Cerrahpasa Medical School, Istanbul University - Cerrahpasa, Cerrahpasa, Turkey; ${ }^{6}$ Nottingham University Hospitals, Nottingham, United Kingdom; ${ }^{7}$ Hospital for Sick Children and University of Toronto, Toronto, Canada; ${ }^{8}$ The Cleveland Clinic, Cleveland, United States of America; ${ }^{9}$ Children's Hospital Colorado, Aurora, United States of America; ${ }^{10}$ Roche Products Ltd, Mississauga, Canada; ${ }^{11}$ F. Hoffmann-La Roche Ltd., Basel, Switzerland; ${ }^{12}$ Roche Products Ltd, Welwyn Garden City, United Kingdom

Background: Low immunoglobulin (Ig) levels can occur after rituximab treatment, but the clinical significance is not completely understood. Not all patients (pts) who develop low Ig levels after rituximab are at an increased risk of serious infection (SI), but factors such as pre-existing low lg levels, prior biologic therapies, history of SI and other disease and age-related factors may increase the risk.

Objectives: To assess the risk of $\mathrm{SI}$ in pediatric pts with prolonged low IgG or IgM serum concentrations following rituximab treatment for GPA or MPA in a global clinical trial.

Methods: In the Phase 2a PePRS study (WA25615), pts aged $\geq 2$ to $\leq 18 \mathrm{yrs}$ with GPA or MPA received 4 weekly intravenous rituximab infusions of $375 \mathrm{mg} / \mathrm{m}^{2}$ body surface area and concomitant oral glucocorticoid taper. After 6 months, pts could receive further rituximab and/or other immunosuppressants at the investigator's discretion during a minimum 12-month follow-up phase. Pts with IgG/lgM levels below age-specific reference ranges at baseline were excluded. Ig levels were measured every 4-12 wks. SI occurrence was assessed during/after low $\lg \mathrm{G}$ or IgM. Prolonged low Ig was defined as IgG or IgM levels < lower limit of normal (LLN) reference range for age for $a \geq 4$-month period.

Results: All 25 pts completed 4 weekly rituximab infusions and the 6-month Remission Induction Phase; $24 / 25$ pts completed $\geq 18$ months of follow-up. 17 pts received additional rituximab treatment on or after Month 6. 11 pts received concomitant immunosuppressants (cyclophosphamide, azathioprine, mycophenolate mofetil) during the study. All pts had a decrease in IgG and IgM mostly after the first rituximab infusion. There was no consistent trend in IgG or IgM levels over time and no clear relationship between low IgG or IgM levels and the number of follow-up rituximab treatments. 18 pts (72\%) had prolonged low $\lg G \geq 4$ months, of whom 5 had IgG levels < LLN at screening and/or baseline; in $7 \mathrm{pts}$, IgG levels returned to within normal range by study end. During or after prolonged low IgG, 6/18 pts experienced a total of $7 \mathrm{SIs}$. Three pts received treatment with intravenous Ig. 19 pts $(76 \%)$ had prolonged low IgM, of whom 5 had IgM levels < LLN at screening and/or baseline. During or after prolonged low IgM levels, 6/19 pts experienced a total of $8 \mathrm{SIs}$. There were no deaths or study discontinuation due to SI. All pts with prolonged low IgG or IgM had past and/ or concomitant treatment with steroids and/or immunosuppressants as potential contributory factors. Analysis of SI onset in relation to timing of low Ig was limited due to protocol-defined time points for Ig assessments.

Conclusion: In pediatric pts with GPA/MPA treated with rituximab, there was no consistent pattern in IgG or IgM levels over time. The majority of pts with prolonged low IgG or IgM did not experience any SIs; no increase in the number of SIs was observed over time or with multiple rituximab treatments. While no firm conclusions can be made on a possible relationship between prolonged low lgG or IgM and risk of SI following rituximab due to study limitations (low pt numbers, lack of placebo comparator), these observations are consistent with the known rituximab safety profile in adult pts with GPA/MPA.

Disclosure of Interests: Simone Melega Shareholder of: F. Hoffmann-La Roche, Employee of: F. Hoffmann-La Roche, Paul Brogan Grant/research support from: Roche, Novartis, SOBI, Chemocentryx, Novimmune, Consultant of: Roche, SOBI, UCB, Novartis, Speakers bureau: Roche, SOBI, UCB, Novartis, Gavin Cleary Speakers bureau: AbbVie, Aimee Hersh: None declared, Ozgur Kasapcopur: None declared, Satyapal Rangaraj: None declared, Rae Yeung Consultant of: AbbVie, Novartis, Speakers bureau: AbbVie, Novartis, Andrew Zeft: None declared, Jennifer Cooper Employee of: Genentech, Inc., Pooneh Pordeli Shareholder of: Roche, Employee of: Roche, Petra Kirchner Shareholder of: Roche, Employee of: Roche, Patricia Lehane Shareholder of: Roche, Employee of: Roche DOI: 10.1136/annrheumdis-2020-eular.750

\section{SAT0504 \\ STING-ASSOCIATED VASCULOPATHY WITH ONSET IN INFANCY (SAVI SYNDROME) CAN MIMIC JUVENILE IDIOPATHIC ARTHRITIS.}

M. Lopez Corbeto ${ }^{1}$, E. Moreno Ruzafa ${ }^{1,2}$. ${ }^{1}$ Hospital Universitari Vall d'Hebron, Pediatric Rheumatology, Barcelona, Spain; ${ }^{2}$ Hospital Universitari Vall d'Hebron, Barcelona, Spain

Background: STING-associated vasculopathy with onset in infancy (SAVI syndrome) can mimic Juvenile Idiopathic Arthritis.
Objectives: The aim of this study is to describe a detailed cohort of patients with SAVI syndrome and highlight the similarity, in some cases, of the phenotype of this disease with Juvenile Idiopathic Arthritis.

Methods: 3 patients diagnosed with SAVI syndrome from the institution Hospital Universitari Vall d'Hebron were recruited. Written informed parental consent was obtained for the use of clinical data and pictures reported. Demographic, clinical, analytical, lung function and previous and current treatment are described.

Results: Patient 1, a 11-year-old boy, was identified to carry a de novo p.V155M mutation in TMEM173. He presented at first month of life with recurrent bronchial infection and skin vasculitis lesions in nose, cheeks and toes. Arthritis affected hands, toes and knees but no erosions were found at X-Ray. Fever was not reported. High-resolution computed tomography (HRCT) of the lungs identified a nonspecific interstitial pneumonia (NSIP) and a lung biopsy showed lymphoid hyperplasia. Elevated inflammatory markers were reported and rheumatoid factor (RF), ACPA antibodies and antinuclear antibodies (ANA) were also positive. At the age of 6 years Ruxolitinib (RX) was introduced at the initial dose of $5 \mathrm{mg}$ twice daily with an improvement of skin disease and lung function. Arthritis was well controlled and RX was well tolerated.

Patient 2, a 17-year-old girl, was identified to carry a de novo p.V155 mutation in TMEM173. She presented at the age of 3 with a severe polyarthritis of large and small joints. No fever, skin or respiratory symptoms were reported at the beginning of the disease. Laboratory tests were positive for RF and ACPA antibodies. She was diagnosed with Polyarticular JIA and was treated with steroids and Methotrexate without improvement. Few months later she reported dyspnoea with recurrent bronchial infections. HRCT showed NSIP and lymphoid interstitial pneumopathy was found at the lung biopsy. RX was initiated at the age of 17 years but at this time lung fibrosis was stablished. Moreover, $\mathrm{RX}$ was not well tolerated due to headache. She requires continuous domiciliary oxygen and has been included to lung transplant.

Finally, patient 3, a 29-year-old man, was recently diagnosed with a de novo p.V155 mutation in TMEM173. He presented at the age of 7 years with symmetrical polyarticular arthritis after a bronchial infection that course with fever. No skin manifestations were objectified. Autoimmune lab test was positive for RF, ACPA, and ANA. With the diagnosis of Polyarticular JIA he received different treatments with no response. Due to recurrent bronchial infections a HRCT was performed showing an ILD at bases and follicular bronchiolitis with NSIP pattern in a lung biopsy. Functional tests were worsening without any response to different treatments. SAVI syndrome was suspected, and genetic test was performed with positive result. $\mathrm{RX}$ was initiated but compliance was not good

Conclusion: SAVI syndrome is a rare monogenic autoinflammatory disease with few cases reported in the literature. Disease phenotype could be different in every patient, with no presence of skin vasculitic lesions or fever. Patient 2 and 3 , in contrast with patient 1 , had severe articular and lung manifestations with no skin involvement. Furthermore, lab tests were positive for RF and ACPA and were misdiagnosed as JIA so genetic test was performed later in the follow-up. Being aware of the distinct phenotype of the disease could help the clinicians to make a PRONTO diagnostic and reassess the patients with these presentations that not respond well to conventional treatments.

References:

[1] Liu Y, et al. Activated STING in a vascular and pulmonary syndrome. N Engl J Med. 2014 Aug 7;371(6):507-518.

Disclosure of Interests: None declared

DOI: 10.1136/annrheumdis-2020-eular.5819

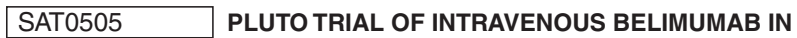 PAEDIATRIC PATIENTS WITH CHILDHOOD-ONSET SYSTEMIC LUPUS ERYTHEMATOSUS (CSLE): PATIENT RESPONSES OVER TIME}

N. Ruperto ${ }^{1}$, L. Mccann ${ }^{2}$, S. Takei ${ }^{3}$, C. Pilkington ${ }^{4}$, D. Bass ${ }^{5}$, B. $\mathrm{Ji}^{6}$, A. Hammer $^{5}$, M. Okily ${ }^{6}$, G. Eriksson ${ }^{5}$, H. Quasny ${ }^{7}$, H. Brunner ${ }^{8} .{ }^{1}$ Istituto Giannina Gaslini, Genoa, Italy; ${ }^{2}$ Alder Hey Children's Hospital, Liverpool, United Kingdom; ${ }^{3}$ Kagoshima University, Kagoshima, Japan; ${ }^{4}$ Great Ormond Street Hospital, London, United Kingdom; ${ }^{5}$ GlaxoSmithKline, Collegeville, United States of America; ${ }^{6}$ GlaxoSmithKline, Uxbridge, United Kingdom; ${ }^{7}$ GlaxoSmithKline, Research Triangle Park, United States of America; ${ }^{8}$ Cincinati Children's Hospital, Cincinnati, United States of America

Background: Belimumab (BEL) is a human monoclonal antibody that specifically inhibits B-cell activating factor (BAFF). PLUTO is an ongoing trial evaluating efficacy and safety of intravenous (IV) BEL in children $\geq 5$ years of age with cSLE. Efficacy, and safety endpoints of PLUTO have been reported; ${ }^{1}$ briefly, numerically more BEL vs PBO pts met the primary and major secondary efficacy endpoints. We present patient (pt) response to BEL over time.

Objectives: To evaluate changes in SLE Responder Index (SRI) 4 and SRI6 responses, and disease activity over 52 weeks, in paediatric pts receiving BEL, or placebo (PBO), plus standard SLE therapy (SST). 
Methods: PLUTO (GSK Study BEL114055, NCT01649765) is a Phase 2, randomised, double-blind, placebo-controlled study. Pts 5-17 years of age with active CSLE were randomised to monthly BEL $10 \mathrm{mg} / \mathrm{kg} \mathrm{IV}$, or PBO, plus SST. Endpoints assessed: SRI4 and SRI6 response rate, mean percentage and absolute change from baseline in Safety of Estrogens in Lupus Erythematosus National Assessment (SELENA)-SLE Disease Activity Index (SLEDAI) and Physicians' Global Assessment (PGA) scores, and percentage of pts with no new British Isles Lupus Assessment Group (BILAG) 1A/2B organ domain scores compared with baseline, all by study visit. The last-observation-carried-forward (LOCF) principle (missing values imputed using the last available non-missing value) was applied to pts who withdrew or received protocol-prohibited medication or a dose of allowable medication that resulted in treatment failure prior to the Week (Wk) 52 visit. Descriptive statistics were used.

Results: A total of 93 pts (94.6\% female, mean [SD] age 14.0 [2.49] years) were randomised for the intention-to-treat (ITT) population: 53 to BEL and 40 to PBO. Mean (SD) BEL and PBO baseline scores were 10.3 (3.34) and 10.4 (3.63) for SELENA-SLEDAI and $1.3(0.43)$ and $1.4(0.42)$ for PGA, respectively. Pt number with at least BILAG 1A/2B organ domain involvement at baseline was 37 (69.8\%) for BEL and 29 (72.5\%) for PBO. SRI4 and SRI6 responses over 52 weeks were mostly numerically higher with BEL than PBO; more BEL than PBO pts were SRI4 and SRI6 responders at Wk 52 (Figure 1). Unadjusted mean (SE) percentage changes from baseline over time in SELENA-SLEDAI and PGA scores generally favoured BEL over PBO, as did unadjusted mean (SE) absolute changes (Figure 2). Wk 52 adjusted mean $(95 \% \mathrm{Cl})$ percentage treatment difference vs PBO was $-4.0 \%(-21.8,13.9)$ for SELENA-SLEDAI and $-6.1 \%(-23.9,11.7)$ for PGA, while Wk 52 adjusted mean $(95 \% \mathrm{Cl})$ treatment difference vs PBO was $-0.7(-2.4,1.1)$ for SELENA-SLEDAI and $-0.1(-0.3,0.1)$ for PGA. Over the study duration, numerically more BEL than PBO pts had no new BILAG $1 A / 2 B$ organ domain scores (Figure 2).

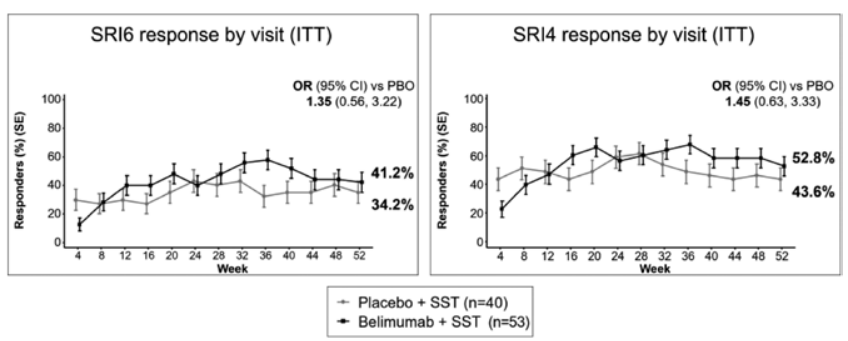

Figure 1. SRI4 and SRI6 response by study visit

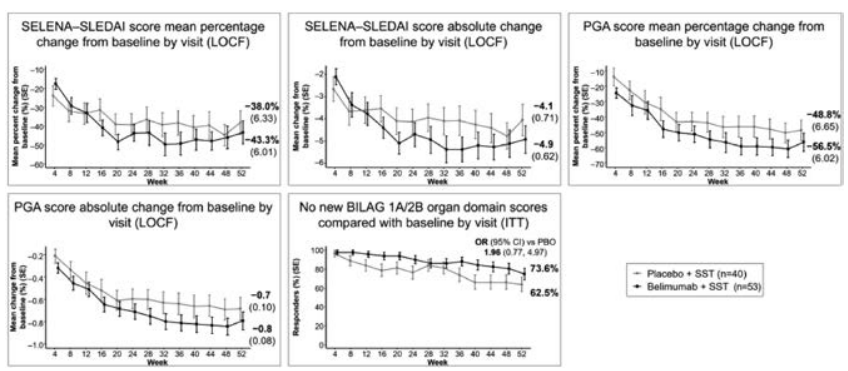

Figure 2. SELENA-SLEDAI and PGA score mean percentage and absolute change from baseline, and no new BILAG 1A/2B organ domain scores compared with baseline, all by study visit

Conclusion: In line with the main analyses performed at Wk $52,{ }^{1}$ further analyses of responses over time in SRI4, SRI6 and disease activity generally favoured BEL over PBO. Combined, these results continue to support the efficacy profile of IV BEL in the treatment of children with cSLE.

References:

[1] Brunner HI, et al. Arthritis Rheumatol. 2018;70(59): 3224-5, Abst. 2867

Acknowledgments: We acknowledge all PLUTO investigators (PRINTO, PRCSG and otherwise affiliated). Study funding: GSK.

Disclosure of Interests: Nicolino Ruperto Consultant of: Ablynx, AbbVie, AstraZeneca-Medimmune, Biogen, Boehringer, Bristol-Myers Squibb, Eli-Lilly, EMD Serono, GSK, Hoffmann-La Roche, Janssen, Merck, Novartis, Pfizer, R-Pharma, Sanofi, Servier, Sinergie, Sobi and Takeda, Liza McCann: None declared, Syuji Takei Grant/research support from: Eisai, Consultant of: Novartis, Bristol-Myers Squibb, Speakers bureau: GSK, Sanofi, Tanabe-Mitsubishi, Novartis, Chugai, Ono, Abbvie, Eli-Lilly, Bristol-Myers Squibb, Clarissa Pilkington: None declared,
Damon Bass Shareholder of: GSK, Employee of: GSK, Beulah Ji Shareholder of: GSK, Employee of: GSK, Anne Hammer Shareholder of: GSK, Employee of: GSK, Mohamed Okily Shareholder of: GSK, Employee of: GSK, Gina Eriksson Shareholder of: GSK, Employee of: GSK, Holly Quasny Shareholder of: GSK, Employee of: GSK, Hermine Brunner Consultant of: Hoffman-La Roche, Novar tis, Pfizer, Sanofi Aventis, Merck Serono, AbbVie, Amgen, Alter, AstraZeneca Baxalta Biosimilars, Biogen Idec, Boehringer, Bristol-Myers Squibb, Celgene EMD Serono, Janssen, Medlmmune, Novartis, Pfizer, and UCB Biosciences, Speakers bureau: GSK, Roche, and Novartis DOI: 10.1136/annrheumdis-2020-eular.4460

\section{SAT0506 \\ MULTIPLE PATTERNS OF 'RESPONSE' TO METHOTHREXATE IDENTIFIED IN A NATIONAL JUVENILE IDIOPATHIC ARTHRITIS COHORT}

S. Shoop-Worrall ${ }^{1,2}$, K. Hyrich ${ }^{2,3}$, L. Wedderburn ${ }^{4,5,6}$, W. Thomson ${ }^{3,7}$ N. Geifman ${ }^{1}$ on behalf of BSPAR-ETN Study, BCRD Study and CLUSTER ${ }^{1}$ The University of Manchester, Centre for Health Informatics, Manchester, United Kingdom; ${ }^{2}$ The University of Manchester, Centre for Epidemiology Versus Arthritis, Manchester, United Kingdom; ${ }^{3}$ Central Manchester University Hospitals NHS Foundation Trust, Manchester Academic Health Science Centre, NIHR Manchester Musculoskeletal BRC, Manchester, United Kingdom; ${ }^{4}$ University College London, Centre for Adolescent Rheumatology Versus Arthritis, GOS Institute of Child Health, London, United Kingdom; ${ }^{5}$ Great Ormond Street Hospital NHS Foundation Trust, Paediatric Rheumatology, London, United Kingdom; ${ }^{6}$ NIHR Great Ormond Street Hospital Biomedical Research Centre, London, United Kingdom; ${ }^{7}$ The University of Manchester, Centre for Genetics and Genomics Versus Arthritis, Manchester, United Kingdom

Background: Disease activity following treatment for JIA is currently understood in terms of 'response' or 'non-response'. This state is usually defined using composite measures such as the ACR Pedi scores or cut-offs on the Juvenile Arthritis Disease Activity Scores (JADAS). However, response is a complex state and it is likely that separate, identifiable clusters of children and young people (CYP) have different, varying levels of response across the individual measures of JIA disease activity. Identifying these clusters may facilitate stratified medicine in JIA. Objectives: To identify clusters of CYP with distinct patterns of change across the individual JADAS components following MTX initiation for JIA.

Methods: MTX-naïve CYP enrolled into the MTX cohorts of the British Society for Paediatric and Adolescent Rheumatology Etanercept Cohort Study or the UK Biologics for Children with Rheumatic Diseases registers before January 2018 were selected. JADAS components (active joint count to 71, physician globa assessment $(0-100 \mathrm{~mm})$, parent global evaluation $(0-100 \mathrm{~mm})$ and $\mathrm{ESR}(\mathrm{mm} / \mathrm{hr})$ ) were collected at MTX start and at (approximately) 6- and 12-month follow-ups. Outcomes were Log1p transformed for analysis and all outcome data were censored following start of a biologic. CYP were excluded if they had clinically inactive disease at MTX initiation, initiated a biologic within a month of MTX or had no available JADAS data at any time point.

Multivariate group-based trajectory models explored MTX response clusters over the first year following MTX initiation using censored-normal models. Linear, quadratic and cubic polynomials were tested, with one to ten trajectories tested within each polynomial group. Optimal models within each polynomial group were selected using Bayesian Information Criteria, after excluding those with groups representing $<1 \%$ of the cohort, average posterior probability for assigned group $<70 \%$ or relative entropy $<0.5$.

Results: Of 657 CYP, the majority were female (69\%) and of white ethnicity (85\%) with RF-negative polyarticular JIA the most common disease category (33\%).

The optimal model identified multiple patterns of disease activity following MTX initiation, with greater complexity than the traditional 'response' or 'non-response' paradigm. Although there were no substantial differences in ESR trajectories between the groups, there were differences in initial disease severity and speeds of improvement across active joint counts, physician and parental global assessments over time. In addition, individual JADAS components did not always change in parallel over time, even within the same cluster of CYP.

Conclusion: There are multiple patterns of disease activity following MTX initiation for CYP with JIA. This suggests that a simple response/non-response analysis at a single time point may be inadequate. Understanding clinical or biological factors associated with these clusters could facilitate stratified medicine in JIA.

Acknowledgments: The CLUSTER consortium is supported by contributions of its industry partners, currently Pfizer, AbbVie UCB, GSK, and Sobi

Disclosure of Interests: Stephanie Shoop-Worrall: None declared, Kimme Hyrich Grant/research support from: Pfizer, UCB, BMS, Speakers bureau: Abbvie, Lucy Wedderburn Speakers bureau: Pfizer, Wendy Thomson: None declared, Nophar Geifman: None declared

DOI: 10.1136/annrheumdis-2020-eular.3241 